\title{
Entwurf einer funktionalistischen Religionspublizistik
}

\author{
von Helmut M. Artus
}

Obwohl die Religionspublizistik in den letzten Jahren wachsenden Raum einnimmt, was sich vor allem in einer eigenen Zeitschrift dokumentiert, ist ihr Zustand als Wissenschaft nach wie vor desolat. Wer sich von der "Communicatio Socialis" (CS) - neben ihren anderen Zwecken - ein Forum religionspublizistischer Forschung erhofft hatte, mußte sich - zumindest bisher - enttäuscht sehen. Dieser unbefriedigende Zustand ist $\mathrm{m}$. E. hauptsächlich auf vier Faktoren zurückzuführen:

1. Religionspublizistik wird offenbar von vielen Autoren noch mehr als religiöse Publizistik denn als Wissenschaft von dieser aufgefaßt, allenfalls als Hilfswissenschaft für religiöse Kommunikatoren. ${ }^{1}$ Die Rollentrennung zwischen Christ (bzw. Seelsorger, Missionar, religiöser Kommunikator usw.) und Publizistikwissenschaftler ist oft nur mangelhaft oder gar nicht vollzogen. Damit ist eine Grundvoraussetzung für Wissenschaft, die Distanz zwischen Subjekt und Objekt der Erkenntnis, nicht erfüllt ${ }^{2}$;

2. eine theoretische Fundierung des Sachgebietes ist noch nicht geleistet (bezeichnenderweise erschien in der CS bisher lediglich ein, zudem wenig befriedigender Aufsatz mit diesbezüglicher Thematik, der weder eine Methode noch einen theoretischen Ansatz bietet); die Stellung der Religionspublizistik innerhalb der gesamten Publizistikwissenschaft ist nicht definiert. Vor allem fehlen Konzeptionen, die den Ubergang vom deskriptiven zum theoretischen Stadium dieser Teilwissenschaft ermöglichen;

3. die Religionspublizistik versteht sich offenbar überwiegend noch nicht als Sozialwissenschaft und kann von daher noch keinen theoretischen Bezugsrahmen für die mit zahlreichen ad-hoc-Methoden gewonnenen empirischen Daten bereitstellen;

4. da Religionspublizistik heute meist noch interessengebunden betrieben wird, beschäftigt sie sich fast notwendigerweise nur mit religiöser Publizistik aus dem christlichen Bereich; auch von dieser Beschränkung her bestehen z. Zt. keine Aussichten, zu umfassenderen Aussagen über das Phänomen „religiöse Publizistik“ zu kommen.

Vom Grundverständnis der Religionspublizistik als spezieller Publizistik und damit als Sozialwissenschaft her können wir die Aufgaben skizzieren, die gelöst werden müssen, um die Religionspublizistik als Wissenschaft konstituieren zu können. Das folgende Schema gibt zugleich einen groben Ưberblick über die thematische Gliederung der Arbeit, die versuchen will, einen Beitrag zur Lösung der angerissenen Probleme zu leisten:

1. Zieldefinition einer theoretisch ausgerichteten sozialwissenschaftlichen Religionspublizistik;

Helmut M. Artus studiert Soziologie und Publizistik an der Universität Münster; er plant eine Abschlußarbeit über Theorie und Realität in den Sozialwissenschaften. 
2. Skizzierung einer Metbode zur Aufbereitung empirisch-deskriptiver Fakten für eine (funktionalistische) Theorie;

3. Klärung der Bedeutung des Transzendenten für eine Sozialwissenschaft;

4. Skizzierung eines Forschungsansatzes, der für die gesamte Publizistikwissenschaft anwendbar ist und somit zur Einheit des Fachs beiträgt, und d. h.: zur Bildung einer allgemeinen Publizistischen Theorie;

5. Definition des Forschungsbereichs.

\section{Zieldefinition und Methode}

Wenn wir Religionspublizistik jenseits von spezifischen Gruppeninteressen als Sozialwissenschaft betreiben wollen, stellt sich als erstes die Frage nach einer wissenschaftlich orientierten Definition der Erkenntnisziele. Wie wir sehen werden, hängt damit die Frage der Methodik aufs engste zusammen.

Als höchstes Ziel von Wissenschaft wird allgemein die Aufstellung gültiger Theorien angesehen, $d$. h. begrifflicher Konstruktionen, die die Elemente ihres Objektbereichs unter dem Aspekt kausaler Zusammenhänge ("wenn-dann-Aussagen“) organisieren. Kausalität bezeichnet dabei ein Ausschließlichkeitsverhältnis zwischen einer spezifischen Ursache und einer spezifischen Wirkung.

Im (sozialwissenschaftlichen) Neopositivismus wurden große Anstrengungen unternommen, eine Methodik zu entwickeln, mit Hilfe derer diese Zusammenhänge (möglichst) objektiv und intersubjektiv nachprüfbar aufgedeckt werden können, jenseits der möglicherweise von ideologischem Vorverständnis belasteten Subjektivität des Forschers. Bei allem Bemühen um Objektivität und Ideologiefreiheit versäumte der Neopositivismus aber, das - gerade in der Soziologie - naheliegende quasi-ideologische Vorverständnis des Kausalitätsbegriffs zu reflektieren.

Dem herkömmlichen kausalwissenschaftlichen Verständnis mit der festen Relation zwischen einer Ursache und ihrer spezifischen Wirkung liegt unausgesprochen und unreflektiert die Prämisse zugrunde, der Gesichtspunkt der Betrachtung von Bewirkungsprozessen sei logisch vorgegeben: von daher sind alle anderen Wirkungen, die von derselben Ursache des Kausalzusammenhanges gezeitigt werden, lediglich Nebenwirkungen oder Begleiterscheinungen.

Nun hat Niklas Luhmann ${ }^{4}$ überzeugend aufgewiesen, daß die Bezugsgesichtspunkte logisch beliebig gewählt werden können, daß also keine starre Kausalrelation zwischen Ursache und Wirkung besteht. Damit treten die Nebenwirkungen logisch gleichberechtigt neben die bisher in ihrer Exklusivität bzw. Dominanz gesehene Hauptwirkung, die "gemeinte Wirkung", den "Zweck", durch dessen Beibehaltung der (traditionelle) Positivismus zeigt, daß er noch dem „subjektiv gemeinten Sinn“ der Hermeneutik verbunden ist und letztlich einer verstehenden Soziologie, die überwunden zu haben er vorgibt, verhaftet bleibt. Damit kann auch die Objektivität seiner Methoden nicht zu objektiven, wertfreien Aussagen führen, da unbegründet und unbewußt nur Teile der jeweiligen fachlichen Wirklichkeit und ihrer Zusammenhänge thematisiert werden. Damit aber setzt sich der Positivismus gerade dem berechtigten Vorwurf aus, den er selbst anderen wissenschaftlichen Richtungen gegenüber erhebt: dem Vorwurf der Ideologie. Denn von seinem unkritischen (angeblich objektiven) Ansatz her ist er letztlich nicht in der Lage, soziale Phänomene in ihrer vollen sozialen Wirkung zu fassen: Die wirkliche Problematik sozialer Zusammenhänge muß ihm notwendigerweise verborgen bleiben. 
Dazu ein Beispiel: Saxer ${ }^{5}$ spricht über die soziale Bedeutung von Kommunikationsprivilegien, speziell über solche der Kirche; auf der einen Seite ermöglichen sie aufgrund besserer Information ein wirkungsvolleres Handeln der Rezipienten, auf der anderen Seite aber gewinnen die Kommunikatoren einen zusätzlichen sozialpsychologischen Sondereinfluß, wodurch ihre Machtposition verstärkt wird. „Kirchliche Kommunikationsprivilegien, ... zu verlangen, den damit unweigerlich verbundenen Machtaspekt dabei aber zu übersehen, verrät... Blindheit bezüglich der elementaren Sozialzusammenhänge von Kommunikation. ${ }^{* B}$ Im Neopositivismus wurde diese Blindheit zum methodischen Prinzip erhoben. Und so gelangt unter der Hand wieder eine starke Wertung in den streng "wertfreien " Positivismus, allein dadurch, daß er eine logisch nicht gerechtfertigte Auswahl aus der Vielfalt seiner Forschungsaspekte trifft.

Hier ist der Punkt, an dem sich Methode und (außerwissenschaftliches) Interesse berühren: In dem Augenblick, in dem sich eine Wissenschaft oder ein Wissenschaftszweig einer Interessengruppe (etwa der Kirche) verpflichtet weiß, setzt sie die Breite ihres Forschungsansatzes aufs Spiel: Der Forschungsaspekt, der Gesichtspunkt, unter dem kausalwissenschaftliche Forschung betrieben werden soll, ist damit vorgegeben, zumindest stark vorbelastet.

Ziel einer sich objektiv gebenden Wissenschaft kann es aber nicht (nur) sein, die soziale Realisierung subjektiv gemeinten Sinns, das „Bewirken einer Wirkung" (Luhmann) und deren Nebenwirkungen und Störfaktoren zu untersuchen. Vielmehr muß der Forscher frei sein zur selbständigen Thematisierung der nichtbeabsichtigten bzw. latenten Folgen. Eine Wissenschaft, die keine kritische Distanz zu ihrem Objekt hat und - ohne diesen Vorgang zu reflektieren - „verwertbares ${ }^{*}$ Wissen liefert, stellt sich in den Dienst des Bestehenden, ohne dieses in seinem Funktionszusammenhang zu durchschauen. Die Funktion einer „ideologiefreien “ Sozialwissenschaft ist damit die der Stabilisierung der bestehenden sozialen und politischen Verhältnisse - und damit selbst eine politische, ungewollt und unbewußt also eine ideologische, insofern sie den status quo stabilisiert.

Soziologie und Publizistik sind noch nicht in einem Stadium, in dem sie wesentliche verwertbare Ergebnisse liefern könnten; sollen sie trotzdem "positive“ Funktionen erfüllen, und soll andererseits einem ideologisch verengten Ansatz vorgebeugt werden, so können (müssen) sie ihre Aufgabe sehen in Aufklärung und Kritik, in einem Beitrag zur Befreiung des Menschen von (unbewußten) sozialen Zwängen. Für ihre Methode bedeutet das: Übergang zur funktionalen Analyse.

Durch den herkömmlichen kausalwissenschaftlichen Ansatz ist beinahe die Gewähr gegeben, daß keine unerwünschten Forschungsergebnisse gezeitigt werden können: zwar negative (Störfaktoren, Fehler, Schwächen usw.), die aber - als Anlässe zu Korrektur und Perfektionierung - wieder positiv (weil verwertbar) sind, um den prozessualen Kausalzusammenhang zu stabilisieren. ${ }^{7}$ Unerwünscht, weil grundsätzlich negativ für den status quo, sind solche kritischen Ergebnisse, die nicht als feed back eines korrigierenden Regelkreises fungieren, sondern eine ganz anders geartete Problematik thematisieren, die u. U. geeignet sein könnte, den Prozeß als solchen von seinen "Nebenfunktionen " her in seiner Berechtigung infragezustellen und damit Gruppen- oder Einzelinteressen zu tangieren. ${ }^{8}$ Durch den streng kausalwissenschaftlichen Ansatz begibt sich also der Wissenschaftler der Möglichkeit, kritische Forschung zu treiben. „Kritisch" bedeutet dabei keine Intendierung von Werten, sondern besagt nur eine Erweiterung der möglichen Aussagen über einen Bewirkungszusammenhang, also eine expandierte Fragestellung, die als solche genausoviel oder 
genausowenig methodisch objektiv ist wie die bisherige und genausoviele oder wenige Werte intendiert. -

„Die funktionalistische॰ Analyse kausaler Faktoren befaßt sich... nicht nur mit der Beziehung zwischen Ursachen und Wirkungen. Eine solche Beziehung wird zwar im Ansatz der Analyse vorausgesetzt. Sie dient als methodisches Hilfsmittel, nicht aber als Gegenstand der Feststellung. Die Analyse selbst konzentriert sich entweder auf die Erforschung möglicher Ursachen unter dem Leitgesichtspunkt einer Wirkung oder auf die Erforschung von Wirkungen unter dem Leitgesichtspunkt einer Ursache. "10 Das heißt: die funktionale Analyse setzt zwar ein Bewirkungsverhältnis voraus, löst sich aber von der starren Exklusivität der spezifischen Ursache-WirkungRelation. Vielmehr hat sie zwei Erkenntnisziele:

[1.] Von einer als Bezugspunkt ad hoc festgelegten tatsächlichen oder gewünschten Wirkung (als Bezugsproblem formuliert!) her wird untersucht, welche verschiedenen Ursachen in der Lage sind, diese Wirkung hervorzubringen, dieses Problem zu lösen. Das wesentliche Neue ist, daß nicht mehr nach mehreren ähnlichen Lösungsmöglichkeiten gefahndet wird, sondern daß unter dem Aspekt eines spezifischen Problems mehrere wesensmäßig verschiedene Handlungen als gleichwertige Lösungen erkannt werden können: als funktional äquivalent. Vergleichsgesichtspunkt ist nicht mehr die äußere Ahnlichkeit der Handlungen, das Wesen, sondern ihre Funktion, ihre Leistung zur Lösung bestimmter Probleme. Das bedeutet die Überwindung des ontologischen Verständnisses des Gleichen als Ahnlichem und führt zu der neuen Sicht des Gleichen als Verschiedenem, aber funktional Äquivalentem. Der spezifische Erkenntniswert dieses Ansatzes liegt im Gewinn der Vergleidhbarkeit der unter dem Aspekt des Wesens unvergleichbaren Handlungen.

Luhmann bringt ein instruktives Beispiel: „Die neuere sozialanthropologische Forschung hat... die Frage aufgeworfen, warum in archaischen Sozialordnungen, die nur kleine Stämme, aber keine zentrale politische Herrschaft kennen, die Reibungen zwischen den Stämmen nicht zu völliger wechselseitiger Vernichtung führen. Die Lösung dieses abstrakt gestellten funktionalen Bezugsproblems der Eindämmung von Konflikten liegt in einem weitverzweigten Verwandtschaftssystem, das über die Grenzen der lokalen Lebensgemeinschaften hinausreicht. Die weite Ausdehnung des Verwandtschaftsbewußtseins führt dazu, daß sich in den einzelnen Stämmen größere Kampftruppen schwer zusammenbringen lassen, ohne daß für das eine oder andere Mitglied auf der Gegenseite Verwandte auftauchen würden. Verwandtschaftsloyalitäten verhindern die Festigung eindeutiger Fronten. Sie brechen die Konfliktlinien in mannigfacher Weise und dämmen die Konfliktmöglichkeiten dadurch ein.

Der Sinn einer solchen Analyse besteht nicht nur im Aufdecken des Zusammenhangs einer Ursache und einer Wirkung ... Der Erkenntnisgewinn steckt in den Vergleichsmöglichkeiten: Absolute Monarchie kann nicht mehr, wie es Hobbes schien, als einzig denkbare Lösung des abstrakten Problems der Konfliktskontrolle angesehen werden. Stark aufgesplitterte Loyalitäten erfüllen die gleiche Funktion. "11

Dies bedeutet, daß spezifische Probleme nicht mit spezifischen Mitteln („Strukturen") gelöst werden müssen, sondern daß prinzipiell eine Reihe von Lösungsmöglichkeiten bereitsteht, die unter dem Vergleichsgesichtspunkt, dem funktionalen Aspekt, gleichwertig sind und sich nur in ihren Folgen ("Nebenwirkungen ${ }^{\alpha}$ ) unterscheiden. Nehmen wir ein Beispiel: Zur Lösung des funktionalen Problems "Produktionssteigerung " hat ein Betrieb z. B. folgende Möglichkeiten: (1) Vergrößerung der Belegschaft, (2) Überstunden, (3) Entlassungsdrohung, (4) Lohnerhöhung, 
(5) Anhebung des Arbeitstempos, (6) Automatisierung usw. Die Funktion „Produktionserhöhung" ist gleich, die Folgen sind unterschiedlich. Im speziellen Fall, in der konkreten Planung, wird sich die Entscheidung an diesen Folgen orientieren.

[2.] Der zweite, für uns wichtigere Punkt, ist die Beziehung zwischen einer spezifischen Ursache und ihren Wirkungen. Greifen wir noch einmal das Beispiel des Betriebs auf: Wir sehen von dem konstant gehaltenen funktionalen Aspekt (Produktionssteigerung) ab und gehen von den funktional äquivalenten Möglichkeiten aus. Die differierenden Folgen sind z. B.: (1), (2), (4) und (6) bedingen höhere Ausgaben, (3) und (5) vielleicht Verschlechterung des Betriebsklimas und der Warenqualität, (5) vielleicht auch zudem mehr Krankmeldungen, (6) Krach mit der Gewerkschaft usw.

Es zeigt sich also, daß eine bestimmte Ursache eine Vielzahl von Wirkungen hat, eine Vielzahl von sozialen Funktionen erfüllt. Begrenzt wird deren prinzipielle Unendlichkeit nur durch Interessenlage ${ }^{12}$, Einfallsreichtum und Leistungskapazität des Forschers.

Mit diesem Funktionsverständnis ist die Grundlage für eine funktionalistische Wirkungsforschung gelegt. Trotz einer gewissen (relativen) methodischen Rückständigkeit des Funktionalismus, scheint der Gedankengang, der zur Auffassung der (exklusiven) Kausalität als Sonderfall der Funktion (gegenüber dem bisherigen: Funktion als Sonderfall der Kausalität) führt, so zwingend, daß ein Übergang vom engen kausalwissenschaftlichen Ansatz zum funktionalistischen unabdingbar scheint. ${ }^{13}$

\section{Die Objektivierbarkeit des Transzendenten}

Für die Philosophen mag Gott ${ }^{14}$ tot sein, für die Naturwissenschaftler nicht objektivierbar, da ex definitione der räumlichzeitlichen Erfahrbarkeit entzogen und in den existenziellen Bereich verwiesen, die (modernen) Theologen mögen sich noch um eine Definition bemühen, die dem heutigen (relativ) aufgeklärten Menschen zuzumuten ist - für die Sozialwissenschaftler, und damit auch die Publizisten, ist "Gott" höchste Realität, - Realität im empirischen Sinn des Objektivierbaren.

Die Schwäche der Sozialwissenschaften, die sich mit Religionsphänomenen befassen, liegt bis heute häufig darin, daß sie in den Bereich des Transzendenten verweisen, der für sie nicht mehr objektivierbar ist. In der Begegnung des Menschen mit dem Numinosen oder der "Erfahrung des Heiligen"1s wird eine Realität jenseits der spezifisch fachlichen angenommen, die der Empirie nicht mehr zugänglich ist.

Dieser Sicht liegt eine unhaltbare Vermischung von Kategorien verschiedener Wissenschaften zugrunde, wodurch - auf Grund der Inhomogenität des begrifflichen Apparats - keine Aussagen mit Erklärungswert (im Sinne der Sozialwissenschaften) mehr möglich sind. "Gott" ist keine sozialwissenschaftliche Kategorie, sondern eine theologische, die wir erst noch in unseren Begriffsapparat zu übersetzen haben. Dabei gehen wir von der Erkenntnis aus, daß Wissenschaften mit ihren Begriffs-Systemen, die einer theoretisch-analytischen Sprache angehören, ein je spezifisches Modell der Wirklichkeit entwerfen, in dem nur die je relevanten Faktoren vertreten sind, und in dem alle relevante Realität in den Kategorien genau einer wissenschaftlichen Disziplin auftaucht. Alle Aussagen haben nur dann einen Erklärungswert, wenn sie innerhalb des relativ geschlossenen, homogenen und konsistenten Begriffsschemas gemacht werden können. 
Das heißt: Wir müssen ein sozialwissenschaftliches (bzw. publizistisches) Modell finden, mit dem wir genau den Teil der Chiffre Gott fassen, der für uns relevant ist, wobei relevant besagt: er muß (a) eine Rolle im publizistischen bzw. sozialen Prozeß spielen, und er muß (b) objektivierbar, d. h. empirisch faßbar sein. ${ }^{16}$

Wie sieht nun dieser "Gott der Publizisten" aus, der zum Objekt empirischer Sozialforschung gemacht werden kann? Wir wollen versuchen, eine Definition zu geben:

Die Leerformel DEUS PUBLICISTICUS ist ein Komplex von Werten und Normen, der durch die Androhung einer unausweichlichen (positiven bzw. negativen) Sanktionierung konformen bzw. abweichenden Handelns und Denkens die Autorität seiner von religiösen Kommunikatoren gesetzten variablen Inhalte (je spezifische Werte und Normen) begründet und der imstande ist, auf soziale Einstellungen und Handlungen zu wirken (und damit soziale Funktionen zu erfüllen) und der ferner als Variable im Wirkungsfeld der sozialen Kommunikation den Prozessen der Spiegelung und Prägung ausgesetzt ist.

Diese Definition wird vervollständigt durch spezifisch religiöse Beigaben (etwa den Begriff "Gott ${ }^{*}$, den Verweis ins Transzendente, die betonte Irrationalität, den Offenbarungscharakter usw.), die jedoch für die funktionalistische Analyse nicht mehr sind als Accessoires. Ausgehend vom Äquivalenzfunktionalismus, können wir - von den wesensmäßigen Specifica abstrahierend - Querverbindungen zu anderen speziellen Publizistiken jenseits des religiösen Bereichs ziehen, die die gleichen funktionalen Merkmale aufweisen. Von hierher ist die Konstruktion von Theorien zwischen den einzelnen partiellen Theorien und der umfassenden publizistischen Theorie des gesamten Fachbereichs denkbar, etwa in der Form einer Theorie totalitärer Systeme, in der wesensmäßig so unterschiedliche Phänomene wie etwa Kirche, nicht-religiöse weltanschauliche Gruppen, Diktaturen usw. unter denselben funktionalen Aspekten subsumiert werden können.17

\section{Religionspublizistik und Systemtbeorie}

Diese methodischen Erwägungen leiten bereits über zum Bereich der Theorie, und unsere Aufgabe wird es nun sein, einen möglichen theoretischen Bezugsrahmen aufzuzeigen, der die Fülle empirischer Daten organisiert, die mit Hilfe der funktionalen Analyse gewonnen werden, der zudem der Einheit des Fachs gerecht wird und der schließlich die Nähe der Soziologie berüdksichtigt. Wir können damit den Problemund Aufgabenbereich der Religionspublizistik nicht mehr einfach auf die Formel der „Erforschung und Beschreibung der religiösen Determinante im publizistischen Geschehen" bringen, wie Prakke und Stoll noch meinen, oder auf die Formel der "religiösen Bedingtheit publizistischer Aussage und ... der publizistischen Bedingtheit religiöser Aussage ${ }^{\text {“18 }}$

Ein Ansatz, der der Einheit der Soziologie Rechnung trägt und dessen Anwendbarkeit auf die Publizistik Rühl19 nachgewiesen hat, ist der systemtheoretische, der im folgenden zugrundegelegt werden soll. Wie Luhmann ${ }^{20}$ zeigt, ist eine deutliche Trennung $\mathrm{zwischen} \mathrm{(funktionalistischer)} \mathrm{Methode} \mathrm{und} \mathrm{Theorie} \mathrm{möglich} \mathrm{und} \mathrm{nötig.} \mathrm{Das}$ bedeutet für uns:

1. eine Methode kann nicht nur nacheinander, sondern auch gleichzeitig verschiedene Theorien hervorbringen; und 
2. die Theorien brauchen nicht unbedingt den gleichen Abstraktionsgrad zu besitzen wie die Methode.

Mit der Entscheidung für eine Methode ist also noch keine Entscheidung für eine Theorie präjudizizert.

Von unserem sozialwissenschaftlichen Ansatz her können wir Kirche (bzw. Religion) als System verstehen, d. h. als einen durch subjektiv gemeinten Sinn konstituierten Zusammenhang von sozialen/kommunikativen Handlungen. Einheiten des Systems sind also nicht Individuen, sondern Handlungen; die Akteure sind Individuen als Rollenträger: Damit ist die Konsequenz aus den Tatsachen gezogen, daß Individuen in verschiedenen Systemen unterschiedlich, aber je spezifisch, handeln; daß je spezifische Erwartungen an sie herangetragen werden; und schließlich: daß nicht Individuen einer funktionalen Analyse unterzogen werden können, sondern Handlungen; nicht Individuen erfüllen Funktionen, sondern Handlungen.

Wenn wir Religionspublizistik (oder allgemein: Publizistik) auf einer systemtheoretischen Konzeption aufbauen wollen, müssen wir von einem erweiterten Ansatzpunkt ausgehen. Es ist nicht so, daß wir die soziologische Systemtheorie nur publizistisch umdeuten müssen, um einen brauchbaren Ansatz zu erhalten, wobei an die Stelle der sozialen Rollen und Handlungen die publizistischen, kommunikativen treten und die eindeutige Trennung gegenüber der Soziologie vollzogen wird. Eine sozialwissenschaftliche Publizistik kann nicht auf kommunikative Prozesse und Inhalte eingeengt werden, da die Wirkung in den Dimensionen des Sozialen auftritt. Damit ergibt sich die Notwendigkeit, diesen Bereich einzubeziehen; und das bedeutet für den theoretischen Ansatz: Wir müssen von einer komplexeren Umwelt des kommunikativen Systems ausgehen, die nicht nur aus publizistischen, sondern aus sozialen Handlungen allgemein besteht.

Im folgenden soll, ohne daß auch nur der Anspruch annähernder Vollständigkeit erhoben wird, der Systembegriff in seinen wichtigsten Zügen skizziert werden. ${ }^{21}$

Ein soziales System ist ein Sinnzusammenhang von Handlungen, der sich gegenüber einer Umwelt nicht dazugehöriger Handlungen abgrenzt und eine relative Invarianz erreicht, so daß die über Systemgrenzen hinausreichenden Bewirkungszusammenhänge das System nicht zwingen, auf jede Anderung der Umwelt mit einer Anderung des Systems zu reagieren. Die Stabilisierung von Systemen geschieht nicht auf der Ebene konkreter Handlungen, sondern auf der Ebene von Verhaltenserwartungen: bestimmte Handlungen werden typisch erwartbar und durch das System garantiert; Systemgrenzen sind Grenzen der Erwartbarkeit von Handlungen.

Durch die Tatsache einer höchst variablen Umwelt - zu der auch das handelnde Individuum als solches gehört! 一, die ständig den Bestand des Systems bedroht, wird das System als Leistungszusammenhang erst problematisch und verständlich: Es muß gewisse (unspezifische!) funktionale Leistungen erbringen, die gegenüber der Umwelt stabil (relativ invariant) gehalten werden können. Das geschieht durch Strukturbildung, durch Konstantsetzung spezifischer Problemlösungen (Handlungen). Die Prozesse der Strukturbildung, und damit Stabilisierung, des Systems geschehen in drei Richtungen:

1. zeitlich: „Die strukturbildenden Verhaltenserwartungen müssen Dauergeltung gewinnen, die auch dann in Kraft bleibt, wenn die Erwartungen im Einzelfall enttäuscht werden. Man ist dann nicht blamiert, wenn man sich trotz der Enttäuschung weiter zu ihnen bekennt."

2. sachlich: „Außerdem müssen die Verhaltenserwartungen zu sachlich konsistenten... Rollen zusammengefügt werden. Dem Rollenträger darf nicht zuviel zugemutet 
werden; vor allem muß ihm eine glaubwürdige Selbstdarstellung seiner Person in den verschiedenartigsten Handlungen möglich sein."

3. sozial: "Und schließlich müssen die Verhaltenserwartungen in gewissem Umfange institutionalisiert werden ... ${ }^{{ }_{222}}$, d. h. die Rollen werden von der konkreten Person abgelöst; die hier fixierten Verhaltenserwartungen werden künftig an jeden Inhaber derselben Position gerichtet, sie bleiben sogar bestehen, wenn die Position einige Zeit nicht besetzt ist.

\section{Objektbereich der Religionspublizistik}

Wir können nun versuchen, den Objektbereich der Religions- (bzw. Kirchen-) publizistik zu umreißen:

1. Religion (bzw. Kirche) als System; dazu die Systemumwelt, insofern Wirkungsanstöße aus ihr kommen oder sich in ihr fortsetzen; außerdem die Interdependenz zwischen System und Umwelt;

2. die Inhalte religiöser Kommunikation, also die religiöse Publizistik ${ }^{23}$;

3. Austauschphänomene $\mathrm{zwischen} \mathrm{religiösem} \mathrm{und} \mathrm{säkularem} \mathrm{Bereich,} \mathrm{bzw.} \mathrm{zwischen}$ System und Umwelt ${ }^{24}$.

Schließlich sei noch etwas über den Anspruch der Systemtheorie gesagt: ihr Universalitätsanspruch besagt lediglich, daß der Begriff des Systems auf alle Handlungszusammenhänge jedweden Umfangs und jeder Art anwendbar ist, gleich ob formalisiert (z. B. Behörden) oder nicht-formalisiert (z. B. Stammtischrunde). Daneben sind durchaus andere Ansätze möglich, d. h.: die Systemtheorie kann keinen Ausschließlichkeitsanspruch erheben.

Die vorgetragenen Erwägungen, die in der vorliegenden Arbeit am Fall der Religionspublizistik exemplifiziert wurden, können nach Meinung des Autors als Modell für andere spezielle Publizistiken fungieren. Methode und Theorie sind von hinreichender Abstraktheit, um verschiedenartige Erscheinungen zu beschreiben, und in dem Absatz über die Objektivierbarkeit des Transzendenten wurde exemplarisch aufgewiesen, daß und wie eine spezielle Publizistik ihren Objektbereich für ihre Belange umdefinieren kann und muß. Eine Einigung auf diesen Ansatz würde von vornherein eine gezielte Forschung ermöglichen, die die Einheit der Publizistik erreichen könnte.

Anmerkungen:

1. vergl. dazu etwa Franz-Josef Eilers (in: CS, Emsdetten, 1:1968 pp. 1-5): „Die neue Zeitschrift soll solchen Erkenntnissen und Erfahrungen aus dem Gesamtbereich der Publizistik ihre Seiten öffnen, die mittelbar oder unmittelbar für kirchliche publizistische Arbeit bedeutsam sein können. Sie soll durch Beiträge von Fadhleuten auf Probleme und Aufgaben hinweisen und zu ihrer Lösung beitragen, - durch Information, Anregung und Kritik. “ (p. 2)

2. vergl. dazu René König über die gleiche Problematik innerhalb der Religionssoziologie (Die Religionssoziologie bei Emile Durkheim, in: Dietrich Goldschmidt und Joadim Matthes (Hrsg.): Probleme der Religionssoziologie $={ }$ Kölner Zeitsdhrift für Soziologie und Sozialspychologie" (KZfSS), Sonderheft 6, Köln und Opladen 1966², pp. 36-49): „... eine methodisch einwandfreie Beschäftigung mit sozialen Phänomenen muß schnell die außerordentliche Bedeutung erkennen lassen, welche die religiösen Verhaltensweisen in der Entwidklung der menschlichen Gesellschaft immer gespielt haben. Das setzt allerdings voraus, daß die religiösen Phänomene zum Gegenstand einer rein wissenschaftlidhen Forschungsmethodik gemacht werden, die jegliche Teilnahme am Leben 
der Religion im Erkenntnisakt ausschließt, weil Religionssoziologie eben zunächst und vor allem eine Analyse religiöser Phänomene zum Inhalt hat und weder eine Fortsetzung des Lebens / der Religion mit Einmischung anderer Mittel (der Wissenschaft) noch eine soziologische Erweiterung einer notwendig immer dogmatischen Theologie sein kann. Weil er letzteres nicht verstanden hatte, trennte sich Gaston Richard, ein ursprünglicher Mitarbeiter Durkbeims, von diesem. Darum trifft auch der Vorwurf des Dogmatismus einzig Richard (und alle, die wie er denken)...* (p. 36 f.)

3. Henk Prakke und Gerhard E. Stoll: Entwurf zu einer Religionspublizistik, in: CS, 1:1968 pp. 34-37; vergl. dazu auch: Gerhard E. Stoll: Erwägungen zur religiösen Publizistik, in: „Publizistik“, Bremen, 10:1965, pp. 15-21.

4. Niklas Luhmann: Funktion und Kausalität: in: KZfSS, 1962 pp. 617-644; auch: derselbe: Funktionale Methode und Systemtheorie, in: „Soziale Welt", Göttingen, 1964 pp. 1-25: "Vergleichsgesichtspunkte können rein logisch beliebig gewählt werden." (p. 9)

5. Ulrich Saxer: Massenkommunikation als Mittel christlicher Verkündigung, in: CS, 2:1969, pp. $106-118$.

6. ebenda p. 107; Auszeichnung vom Verfasser.

7. vergl. dazu Franz-Josef Eilers a.a.O.: „Sie [die CS] soll durch Beiträge von Fachleuten auf Probleme und Aufgaben hinweisen und zu ihrer Lösung beitragen, - durch Information, Anregung und Kritik. (p. 2)

8. vergl. dazu noch einmal das Beispiel von Saxer.

9. Zur Entwicklung des Funktionsbegriffs siehe Brigitte Steinbeck: Einige Aspekte des Funktionsbegriffs in der positiven Soziologie und in der kritischen Theorie der Gesellschaft, in: „Soziale Welt", 1964, pp. 97-129.

10. Niklas Luhmann: Funktion und Kausalität, a.a.O. p. 628; Auszeichnung vom Verfasser.

11. derselbe: Funktionale Methode und Systemtheorie, a.a.O. p. 7.

12. Hier hält die Subjektivität wieder Ėinzug, aber nicht in der kaschierten Form des herkömmlichen Positivismus; vielmehr ist der Forscher gehalten, Rechenschaft abzulegen: Die Thematik der Analyse wird hinterfragbar.

13. Der funktionalistische Ansatz steht ja nicht im Alternativverhältnis zum kausalwissenschaftlichen, sondern geht über ihn hinaus, beinhaltet ihn aber weiter als Grenzfall.

14. In der vorliegenden Arbeit wird aus naheliegenden Gründen von Kirche und Gott im dristlichen. Verständnis ausgegangen; eine allgemeine Religionspublizistik müßte stattdessen von Religion und einem Transzendenten, Absoluten oder einem religiösen Aquivalent ausgehen, da es Religionen gibt, die ohne dezidierte Gottesvorstellung auskommen.

15. Henk Prakke und Gerhard E. Stoll a.a.O. p. 35.

16. Es sei hier noch darauf hingewiesen, daß dieser Ansatz nichts mit naivem Aufklärertum gemein hat: der theologische "Gott" wird nicht als solcher durch einen sozialwissenschaftlichen ersetzt, sondern nur im Rahmen sozialwissenschaftlicher Aussagen. Über die tatsächliche Existenz wird nichts ausgesagt, weder im positiven noch im negativen, er wird nicht als solcher in den sozialen Prozeß eingemeindet. Wichtig ist, sich stets zu vergegenwärtigen, daß der DEUS SOCIOLOGICUS bzw. PUBLICISTICUS ein theoretisch-analytisches Produkt, ein Modell in einer theoretischen Sprache, ist.

17. Das Verhältnis von Struktur und Funktion ist in keiner Richtung eindeutig, so daß nicht von der Erfüllung bestimmter Funktionen auf die strukturelle Bedingung "Religion" geschlossen werden kann, wie $O$. $H$. von der Gablentz meint: "Integration ist die Funktion von Religion. Umgekehrt: wo diese Funktion der Integration wirklich erfüllt wird, muß der Soziologe von Religion sprechen, auch bei säkularen Ideologien und Riten." (Zitiert nach Joachim Matthes: Religion und Gesellschaft - Einführung in die Religionssoziologie I $=$ rde 279/280, Reinbek 1967, p. 21.) Hier liegen mehrere Mißverständnisse vor: (1) das Exklusivitätsverhältnis von Funktion als Sonderfall der Kausalität; und z. T. daraus resultierend (2) die Eindeutigkeit von Struktur und Funktion; (3) die Verkennung der Möglichkeiten und Erkenntnisabsichten funktionaler Analysen, die es gerade ermöglichen, die gleiche Funktion im Verschiedenen zu erkennen. Bei v. d. Gablentz werden die Specifica von Religiösem und Nicht-Religiösem eingeebnet, die Eigenständigkeit der Phänomene wird geleugnet.

18. beides Prakke/Stoll a.a.O. p. 35.

19. Manfred Rühl: Systemdenken und Kommunikationswissenschaft, in: „Publizistik“, Konstanz, 14:1969, pp. 185-206.

20. Funktionale Methode und Systemtheorie, a.a.O. p. 3 ff. 
21. Wir folgen dabei dem funktional-strukturellen Ansatz von Niklas Luhmann; vgl. dazu opera citata, außerdem: derselbe: Soziologie als Theorie sozialer Systeme, in: KZfSS, 1967, pp. 615-644; außerdem: Manfred Rühl a.a.O.

22. alle drei Zitate aus $\mathrm{N}$. Luhmann: Funktionale Methode und Systemtheorie, a.a.O. p. 17.

23. vergl. dazu H. Prakke/G. E. Stoll a.a.O. p. 36: „..1. säkularisierte Religionspublizistik... 2. allgemein religiöse Publizistik (...); 3. bekenntnisorientierte Publizistik $(\ldots) ; 4$. kirchlich-institutionelle Publizistik (...)."

24. Auch dies ist, wenn auch in anderem Zusammenhang, bercits bei Prakke/Stoll skizziert, doch müßte etwa die Säkularisierung bzw. der ihr entgegengesetzte Prozeß unter theoretischen und methodologischen Aspekten neu definiert und präzisiert werden.

\section{S U M M A R Y}

Religious Communications (Religionspublizistik) are part of social science. Therefore religious communications have to apply all of their objectives into the categories of social science. This is even true for the transcendental area. They have to literate themselves from all group dependence and must try to consider their subjects critically. The right method for this is the functional analysis. Objects of the religious communications science (Religionspublizistik) are religious communications, religion (church) and the interdependencies between religion and the secular field. Religion can be looked upon as a public action system in an environment of social action. Thus, the unity of social communications science and the mutual influence between religion and social environment are respected.

\section{RESUMEN}

El periodismo religioso es una ciencia social. Debe, por lo tanto, establecer todos sus objetos en categorías socio-científicas, aún en el sector de lo trascendente. Debe librarse de adherir a ideas de grupos e investigar críticamente su objeto. El método adecuado es la análisis funcional. Los objetivos de la ciencia de las comunicaciones religiosas son el periodismo religioso, la religión (Iglesia) y las relaciones recíprocas entre la religión y el campo secular. La religión puede ser considerada como un sistema de acción pública en un ambiente de actividad social. Este trabajo examina la unidad de la ciencia de las comunicaciones sociales y la influencia recíproca de la religión y el ambiente. 